\title{
Extended Generalized Riccati Equation Mapping for Thermal Traveling-Wave Distribution in Biological Tissues through a Bio-Heat Transfer Model with Linear/Quadratic Temperature-Dependent Blood Perfusion*
}

\author{
Emmanuel Kengne ${ }^{\#}$, Fathi Ben Hamouda, Ahmed Lakhssassi \\ Department of Computer Science and Engineering, University of Quebec at Outaouais, Gatineau, Canada \\ Email: "kengem01@uqo.ca, ftouh.benhammouda@laposte.net, ahmed.Lakhssassi@uqo.ca
}

Received September 13, 2013; revised October 13, 2013; accetped October 20, 2013

Copyright (C) 2013 Emmanuel Kengne et al. This is an open access article distributed under the Creative Commons Attribution License, which permits unrestricted use, distribution, and reproduction in any medium, provided the original work is properly cited.

\begin{abstract}
Analytical thermal traveling-wave distribution in biological tissues through a bio-heat transfer (BHT) model with linear/quadratic temperature-dependent blood perfusion is discussed in this paper. Using the extended generalized Riccati equation mapping method, we find analytical traveling wave solutions of the considered BHT equation. All the travelling wave solutions obtained have been used to explicitly investigate the effect of linear and quadratic coefficients of temperature dependence on temperature distribution in tissues. We found that the parameter of the nonlinear superposition formula for Riccati can be used to control the temperature of living tissues. Our results prove that the extended generalized Riccati equation mapping method is a powerful tool for investigating thermal traveling-wave distribution in biological tissues.
\end{abstract}

Keywords: Bio-Heat Transfer; Pennes Bio-Heat Model; Temperature-Dependent Blood Perfusion; Thermal Therapy; Extended Generalized Riccati Equation Mapping Method

\section{Introduction}

Heat transfer in biological systems is relevant in many diagnostic and therapeutic applications involving either decrease or increase of temperature and often requires precise monitoring of the spatial distribution of thermal histories that are produced during a treatment protocol [1-9]. Since the pioneering work by Henriques and Moritz [10] and by Pennes [11] on heat transfer in biological system, the problem of heat transfer in biological systems has received renewed attention and has been the focus of considerable research [12-16]. The investigation of such heat transfer problems requires the evaluation of spatiotemporal distributions of temperature and has been traditionally addressed using the Pennes model which is originally designed for predicting temperature fields in the human forearm $[10,11]$. Pennes model of the heat transfer in biological systems accounts for the ability of tissue to remove heat by both passive conduction (diffusion)

*This work was supported by the Natural Sciences and Engineering Research Council of Canada under grants Nos. 7033009.

${ }^{\#}$ Corresponding author. and perfusion of tissue by blood. Here, perfusion is defined as the nonvectorial volumetric blood flow per tissue volume in a region that contains sufficient capillaries that an average flow description is considered reasonable.

After performing a series of experiments which measured temperatures of human forearms, Pennes derived a thermal energy conservation equation called the "bioheat transfer" (BHT) equation or the traditional BHT equation. The generalized one-dimensional Pennes BHT equation can be written in the following form [17-21]

$$
\rho c \frac{\partial T}{\partial t}=k \frac{\partial^{2} T}{\partial x^{2}}-c_{b} \rho_{b} \omega_{b}\left(T-T_{a}\right)+Q_{m}+Q_{p},
$$

where $T\left({ }^{\circ} \mathrm{C}\right)$ is the local tissue temperature (temperature elevation); $t$ and $x$ denote the time and the distance from the skin surface to the body core, respectively; $T_{a}\left({ }^{\circ} \mathrm{C}\right)$ is the arterial blood temperature (ambient temperature of perfusing blood $) ; c_{b}\left(\mathrm{~J} / \mathrm{kg} /{ }^{\circ} \mathrm{C}\right)$ is specific heat of blood; $\rho_{b}$ is the density of blood; $\omega_{b}(\mathrm{ml} / \mathrm{s} / \mathrm{ml})$ is the blood perfusion; $c\left(\mathrm{~J} / \mathrm{kg} /{ }^{\circ} \mathrm{C}\right)$ is the tissue specific heat; $\rho\left(\mathrm{kg} / \mathrm{m}^{3}\right)$ is the tissue density; $k\left(\mathrm{w} / \mathrm{m} /{ }^{\circ} \mathrm{C}\right)$ is 
the tissue thermal conductivity; $Q_{p}\left(\mathrm{w} / \mathrm{m}^{3}\right)$ is the regional heat delivered by the source; $Q_{m}\left(\mathrm{w} / \mathrm{m}^{3}\right)$ is the heat generation in the body, that is, the heat generated by the normal processes in the body, which is usually very small compared to the external power deposition term $Q_{p}$ [22]. When the metabolic heat generation rate ( $Q_{m}=\partial Q /\left.\partial t\right|_{\text {metabolic }}$ where $Q$ is the total heat) is positive, body temperature will rise and when $Q_{m}$ is negative, it will go down [23]; we remember that the total heat $Q$ transported across a flat surface of area $A$ and thickness $\Delta x$ is related to the temperature gradient across the surface $\Delta T$ and the thermal conductivity of the material: $Q=-k A \frac{\Delta T}{\Delta x}$. Equation (1.1) describes the influence of blood flow on the temperature distribution in the tissue in terms of volumetrically distributed heat sinks or sources.

The second term $c_{b} \rho_{b} \omega_{b}\left(T-T_{a}\right)$ in the right hand side (r.h.s.) of Equation (1.1) models the perfusion heat loss and can be the dominant form of energy removal when considering heating processes. Vascular tissues generally experience increased perfusion as temperature increases. The above term is always considered in cases of tissues with a high degree of perfusion, such as liver. Regarding cardiac ablation, the perfusion heat loss is incorporated in some models, but is generally ignored since its effect is negligible [24]. In general, $\omega_{b}$ is assumed to be uniform throughout the tissue. However, its value may increase with heating time because of vasodilatation and capillary recruitment.

Spatially distributed heating occurs in skin exposed to penetrating, dissipative radiation such as microwave, ultrasound, and laser light [25-27]. These heating methods often involve an exponentially decaying power transmission accompanied by reflection at the interface of regions with different electrical properties. For a uniform plane wave incident normally upon the skin surface, with a layer of air included to model the reflection at the skin/air interface, the average absorbed power density $Q_{p}$ is given by: $Q_{r}=\frac{1}{2} \sigma|E|^{2}$, where $E$ and $\sigma$ are the total electric field in the tissue and the electric conductivity, respectively.

Prediction of temperature in tissue models generally assumes a constant-rate (temperature independent) blood perfusion within each tissue and has long been carried out by both analytical and numerical methods based on Equation (1.1). In this case, the temperature rise can be easily predicted by traditional analytical methods based on Equation (1.1) and given boundary conditions, which can either be solved analytically for simple geometries $[28,29]$ or by finite element method or finite-difference method for more realistic, complicated tissue geometry [30,31].

Several experiments have shown that the response of vasculature in tissues to heat stress is strongly temperature-dependent [32]. Obviously, models which include temperature-dependent increases in perfusion are more difficult to solve. The case of a linear temperature-dependence has been described combining analytical simulations [9,33,34]. Lang et al. [33] described an optimization process specially designed for regional hyperthermia of deep-seated tumors in order to achieve desired steadystate temperature distributions. To predict the temperature, they applied a nonlinear three-dimensional heat transfer model based on temperature-dependent blood perfusion. Using linearly implicit methods in time and adaptive multilevel finite elements in space, they were able to integrate efficiently the instationary nonlinear heat Equation with high accuracy. Temperature distributions for two individual patients calculated on coarse and fine spatial grids were compared. Most recently, Partridge et al. [35] presented an inverse analysis procedure based on a coupled numerical formulation first to identify the coefficients of linear and quadratic variations of blood perfusion, and then to investigate temperature distribution at the skin surface for tumor perfusion. Their procedure has the advantage of not requiring the calculation of derivatives or sensitivities, or an initial estimate of these values.

We assume in this work a linear and a quadratic temperature-dependent blood similar to the expression identified respectively by Gowrishankar et al. [36] and Partridge et al. [35]:

$$
\omega_{b}=\alpha_{0}+\beta_{0} T+\gamma_{0} T^{2},
$$

where $\alpha_{0}(\mathrm{ml} / \mathrm{s} / \mathrm{ml}), \quad \beta_{0}\left(\mathrm{ml} / \mathrm{s} / \mathrm{ml} \cdot\left({ }^{\circ} \mathrm{C}\right)^{-1}\right)$, and $\gamma_{0}\left(\mathrm{ml} / \mathrm{s} / \mathrm{ml} \cdot\left({ }^{\circ} \mathrm{C}\right)^{-2}\right)$ are three real parameters. $\alpha_{0}$ represents the baseline perfusion while $\beta_{0} / \alpha_{0}$ and $\gamma_{0} / \alpha_{0}$ are respectively the linear and quadratic coefficients of temperature dependence. The unit employed for coefficient $\alpha_{0}, \quad \beta_{0}$ and $\gamma_{0}$ is such that $\omega_{b} \rho_{b}$ represents mass flow rate of blood per unit volume of tissue. Based on the Pennes BHT Equation (1.1) with the blood perfusion (1.2), we aim in the present work to analytically investigate thermal traveling-wave distribution in biological tissues. We consider only the case of constant spatial heating, that is, the case when $Q_{p}(x, t)=Q_{p}=$ constant.

In our knowledge, there has not yet being reported a scientific work that treats thermal traveling-wave distribution in biological tissues by means of Riccati Equation

$$
\frac{\mathrm{d} u}{\mathrm{~d} \xi}=u^{2}+\tilde{\beta}(\xi) u+\tilde{\alpha}(\xi) .
$$


In Equation (1.3), $\tilde{\beta}(\xi)$ and $\tilde{\alpha}(\xi)$ are two real functions of $\xi$. The Riccati Equation, though a nonlinear Equation, is characterized by the fact of possessing a superposition formula, as it is the case for all linear equations. Moreover, Riccati equation can be linearized; indeed, we can use the Cole-Hopf transformation [37,38] to reduce it to a linear Schrödinger spectral problem. Riccati Equation (1.3) appears in many fields of applied mathematics, in many instances when we can find exact solutions of nonlinear partial differential equations. In mathematical literatures, Riccati Equation (1.3) is known as the only first order nonlinear ordinary differential equation which has no movable singularity, i.e., which possesses the Painlevé property $[39,40]$. As it was shown by Lie et al. [41], Riccati Equation (1.3) admits a (nonlinear) superposition formula

$$
\begin{aligned}
& \tilde{u}(\xi) \\
= & \frac{u_{1}(\xi)\left[u_{3}(\xi)-u_{2}(\xi)\right]+\tilde{k} u_{2}(\xi)\left[u_{1}(\xi)-u_{3}(\xi)\right]}{u_{3}(\xi)-u_{2}(\xi)+\tilde{k}\left[u_{1}(\xi)-u_{3}(\xi)\right]},
\end{aligned}
$$

that is, if $u_{1}(\xi), u_{2}(\xi)$ and $u_{3}(\xi)$ are three solutions of Equation (1.3), and $\tilde{u}(\xi)$ will also be a solution of Equation (1.3), with $\tilde{k}$ being a constant parameter. The nonlinear superposition Formula (1.4) allows us to build a denumerable set of solutions of Riccati Equation (1.3), as soon as three of its solutions are found.

The aim of the present work is to apply Riccati Equation (1.3) to analytically investigate thermal traveling-wave distribution in biological tissues through the BHT model (1.1) with the temperature-dependent blood perfusion (1.2). The rest of the paper is organized as follows. In Section 2, we explore the solutions of Riccati Equation (1.3) in the special case of constant coefficients $\tilde{\beta}(\xi)$ and $\tilde{\alpha}(\xi)$. In Section 3, we apply the generalized Riccati equation mapping method [42] to find solutions to Equation (1.1) with perfusion (1.2). Here, we distinguish the initial processus (temperature at each distance $x$ from the skin surface to the body core at time $t=0)$, instantaneous temperature distribution of biological tissues (temperature at each distance $x$ from the skin surface to the body core at a given time $t$ ), and the steady-state temperature distribution (the temperature of each point $x$ of the biological tissue is constant with respect with time). The results are discussed in Section 4 and summarized in Section 5.

\section{Solutions of Riccati Equation (1.3) in the Special Case of Constant Coefficients $\tilde{\beta}(\xi)$ and $\tilde{\alpha}(\xi)$}

In this section, we explore the possible solutions of Riccati Equation (1.3) in the special case of constant coefficients $\tilde{\beta}(\xi)$ and $\tilde{\alpha}(\xi)$. Let us consider Riccati equation

$$
\frac{\mathrm{d} u}{\mathrm{~d} z}=p+r u+s u^{2}
$$

where $p, r$, and $s$ are three real parameters with $s \neq 0$. One easily verify that transformation

$u=-y^{\prime} /(s y)$ reduces Equation (1.5) into the second order linear ordinary differential equation (ODE) with constant coefficients

$$
\frac{\mathrm{d}^{2} y}{\mathrm{~d} z^{2}}-\frac{r}{s} \frac{\mathrm{d} y}{\mathrm{~d} z}+p y=0 .
$$

It is also well-known that the transformation $u=u_{p}+1 / w$ reduces the Riccati Equation (1.5) into the the below first order ODE as soon as $u_{p}$ is a particular solution of (1.5)

$$
\frac{\mathrm{d} w}{\mathrm{~d} z}+\left[r+2 s u_{p}\right] w+s=0
$$

Integrating Equations (1.6) and (1.7) yields

$$
\begin{aligned}
& y(z) \\
& =\left\{\begin{array}{l}
C_{1} \exp \left[\frac{r+\sqrt{r^{2}-4 s^{2} p}}{2 s} z\right]+C_{2} \exp \left[\frac{r-\sqrt{r^{2}-4 s^{2} p}}{2 s} z\right], \\
\text { if } r^{2}-4 s^{2} p>0, \\
C_{1}+C_{2} \frac{r}{2 s} z, \\
\text { if } r^{2}-4 s^{2} p=0, \\
{\left[C_{1} \cos \sqrt{4 s^{2} p-r^{2}} z+C_{2} \sin \sqrt{4 s^{2} p-r^{2}} z\right] \exp \left[\frac{r}{2 s} z\right]} \\
\text { if } r^{2}-4 s^{2} p<0,
\end{array}\right.
\end{aligned}
$$

$$
\begin{aligned}
& w(z) \\
= & \frac{1}{u\left(z_{0}\right)-u_{p}\left(z_{0}\right)} \exp \left[-\int_{z_{0}}^{z}\left[r+2 s u_{p}(\xi)\right] \mathrm{d} \xi\right] \\
& -s \int_{z_{0}}^{z} \exp \left[\int_{z}^{\eta}\left[r+2 s u_{p}(\xi)\right] \mathrm{d} \xi\right] \mathrm{d} \eta,
\end{aligned}
$$

respectively, where $C_{1}$ and $C_{2}$ are two arbitrary real constants. Expression (1.8) gives the following general solution of Riccati Equation (1.5) 


$$
u(z)= \begin{cases}-\frac{\left(r+\sqrt{r^{2}-4 s^{2} p}\right) C_{1}+\left(r-\sqrt{r^{2}-4 s^{2} p}\right) C_{2} \exp \left[-\frac{\sqrt{r^{2}-4 s^{2} p}}{s} z\right]}{2 s^{2}\left\{C_{1}+C_{2} \exp \left[-\frac{\sqrt{r^{2}-4 s^{2} p}}{s} z\right]\right\}}, & \text { if } r^{2}-4 s^{2} p>0, \\ -\frac{C_{2} r}{s\left[2 s C_{1}+C_{2} r z\right]}, & \text { if } r^{2}-4 s^{2} p=0, \\ -\frac{r C_{1}+C_{2} \sqrt{4 s^{2} p-r^{2}}+\left(r C_{2}-C_{1} \sqrt{4 s^{2} p-r^{2}}\right) \tan \frac{\sqrt{4 s^{2} p-r^{2}}}{2 s} z}{2 s^{2}\left(C_{1}+C_{2} \tan \frac{\sqrt{4 s^{2} p-r^{2}}}{2 s} z\right)}, & \text { if } r^{2}-4 s^{2} p<0,\end{cases}
$$

while expression (1.9) gives the following general solution of Riccati Equation (1.5)

$$
\begin{aligned}
& u(z)=u_{p} \\
& +\frac{\exp \left[\int_{z_{0}}^{z}\left[r+2 s u_{p}(\xi)\right] \mathrm{d} \xi\right]}{\frac{1}{u\left(z_{0}\right)-G_{p}\left(z_{0}\right)}-s \int_{z_{0}}^{z} \exp \left[\int_{z_{0}}^{\eta}\left[r+2 s u_{p}(\xi)\right] \mathrm{d} \xi\right] \mathrm{d} \eta} .
\end{aligned}
$$

Really speaking, solution (1.10) contains only one free parameter, namely, $C_{0}=C_{1} / C_{2}$.

\section{Thermal Traveling-Wave Distribution in Living Tissues}

With the help of Riccati equation, we investigate in this section the thermal traveling-wave distribution in biological tissues through the BHT Equation (1.1) with the temperature-dependent blood perfusion (1.2). In other words, we present in this section the application of the Riccati equation to find analytical traveling-wave solutions of equation

$$
\begin{aligned}
\rho c \frac{\partial T}{\partial t}= & k \frac{\partial^{2} T}{\partial x^{2}}-c_{b} \rho_{b}\left[\alpha_{0}+\beta_{0} T+\gamma_{0} T^{2}\right]\left(T-T_{a}\right) \\
& +Q_{m}+Q_{p}
\end{aligned}
$$

with constant $Q_{p}$. We start by transforming Equation (1.12) into a nonlinear ordinary differential equation by introducing the variable $z$ given by

$$
T(x, t)=u(z), z=a x-v t,
$$

where $a \neq 0$ and $v$ are two arbitrary constants ( $v$ is the wave velocity). Inserting ansatz (1.13) into Equation (1.12) yields

$$
\begin{aligned}
-v \rho c \frac{\mathrm{d} u}{\mathrm{~d} z}= & a^{2} k \frac{\mathrm{d}^{2} u}{\mathrm{~d} z^{2}}-c_{b} \rho_{b}\left[\alpha_{0}+\beta_{0} u+\gamma_{0} u^{2}\right]\left(u-T_{a}\right) \\
& +Q_{m}+Q_{p} .
\end{aligned}
$$

We first apply the generalized Riccati equation mapping method, and then a simple coupling method to find analytical solutions of Equation (1.14).

\subsection{Generalized Riccati Equation Mapping Method for Temperature Distribution in Biological Tissues}

The generalized Riccati equation mapping method assumes that Equation (1.12) admits solutions that can be expressed as a polynomial of degree $m$ in $\left(G^{\prime} / G\right)$ :

$$
u(z)=\sum_{n=0}^{m} A_{n}\left(\frac{G^{\prime}}{G}\right)^{n}, A_{m} \neq 0, m \geq 1,
$$

where $A_{0}, A_{1}, \cdots, A_{m}$ are real constants to be determined and $G$ is solution of the Riccati Equation (1.5). The first step in this method consists of determining $\mathrm{m}$. To determine $m$, we consider the homogenous balance between the highest order derivative and the highest order nonlinear terms. After finding $m$, one substitutes Equation (1.15) into Equation (1.14) and use the fact that $G$ is a solution of Equation (1.5). Then, one collects all terms with the same order of $1 / G^{n}$ and $G^{n}, n=0,1, \cdots, m$. If the coefficients of $G^{n}$ and $G^{-n}$ vanish separately, one will obtain a system of algebraic equations in $A_{0}, A_{1}, \cdots, A_{m}, \quad r, \quad s, \quad p, \quad a$, and $v$ that physical solutions can be found with the help of Mathematica (here, physical solution means solutions of the algebraic system that define physical solutions of Equation (1.1) with perfusion (1.2)). Finally, one uses the solutions of Riccati Equation (1.5) found in the previous section, and goes back in original variables $x$ and $t$ through ansatz (1.13) to obtain travelling wave solution of Equation (1.12).

Let us now turn to the concrete case of Equation (1.5). We start by inserting Equation (1.15) into Equation (1.14). Using the fact that $G$ is a solution of Equation (1.5) and balancing the highest order of derivative term 
and nonlinear term, we get that $m=1$ for a quadratic blood perfusion, and $m=2$ for a linear blood perfusion. So that Equations (1.15) and (1.5) give

$$
\begin{gathered}
u(z)=A_{0}+A_{1}\left(r+\frac{p}{G}+s G\right) \\
u(z)=A_{0}+A_{1}\left(r+\frac{p}{G}+s G\right)+A_{2}\left(r+\frac{p}{G}+s G\right)^{2}
\end{gathered}
$$

for quadratic and linear blood perfusion, respectively. By combining solution (1.10) of Riccati equation and expression (1.16a) and going back to original coordinates $x, t$, and $T$, one easily sees that The steady-state analytical solution to the bio-heat Equation (1.1) with blood perfusion (1.2.) differs with a spatially distributed uniform sink given in Ref. [43].

Case of a quadratic temperature-dependent blood perfusion

Let us start with the case of a quadratic blood perfusion, that is, when $\gamma_{0} \neq 0$. Then Equation (1.14) admits solutions of form (1.16a). Inserting Equation (1.16a) into Equation (1.14) and imposing to the coefficients of $G^{n}$ and $G^{-n}$ to vanish separately, we obtain, after the compatibility requirement, the algebraic system (see (1.17) below)

Here,

$$
\begin{aligned}
& \alpha=-\gamma_{0} c_{b} \rho_{b} ; \beta=c_{b} \rho_{b}\left(\gamma_{0} T_{a}-\beta_{0}\right) ; \\
& \gamma=c_{b} \rho_{b}\left(\beta_{0} T_{a}-\alpha_{0}\right) ; \delta=Q_{m}+Q_{p}+\alpha_{0} T_{a} c_{b} \rho_{b} .
\end{aligned}
$$

It should be noted that condition $v=0$ corresponds to either the initial temperature distribution or the steady- state temperature distribution. Indeed, the initial temperature distribution and the steady-state temperature distribution in biological tissues is obtained by integrating the ordinary differential equations

$$
\begin{gathered}
a^{2} k \frac{\mathrm{d}^{2} T}{\mathrm{~d} x^{2}}-c_{b} \rho_{b}\left[\alpha_{0}+\beta_{0} T+\gamma_{0} T^{2}\right]\left(T-T_{a}\right)+Q_{m}, \quad(1.18) \\
a^{2} k \frac{\mathrm{d}^{2} T}{\mathrm{~d} x^{2}}-c_{b} \rho_{b}\left[\alpha_{0}+\beta_{0} T+\gamma_{0} T^{2}\right]\left(T-T_{a}\right)+Q_{m}+Q_{p},
\end{gathered}
$$

respectively. Substituting ansatz (1.13) with $v=0$ into Eqs. (1.18) and (1.19), we obtain Equation (1.14) in which $v$ is replaced by 0 . It is important to point out that the term $Q_{p}$ is absent in Equation (1.18). Indeed, prior to heating process, there is not any heating source. Thus, for the initial temperature distribution and the steady-state temperature distribution, we will work with $v=Q_{p}=0$ and $v=0$ with $Q_{p}$ any real constant. We notice that in the case of absence of heating sources, the initial temperature coincides with the steady-state temperature fields.

Case of a linear temperature-dependent blood perfusion

Here, we consider the case of a linear blood perfusion, that is, when $\gamma_{0}=0$ and $\beta_{0} \neq 0$. As it is shown above, Equation (1.14) admits solutions of form (1.17). Inserting Equation (1.17) into Equation (1.14) and imposing to the coefficients of $G^{n}$ and $G^{-n}$ to vanish separately, we obtain, after the compatibility requirement, the algebraic system (see (1.20) below)

$$
\begin{gathered}
\qquad\left\{\begin{array}{l}
p v=0, p^{3}\left(2 a^{2} k+\alpha A_{1}^{2}\right)=0,2 a^{2} k+\alpha A_{1}^{2}=0, \\
p^{2}\left[3 a^{2} k r A_{1}-v \rho c A_{1}+\beta A_{1}^{2}+3 \alpha A_{1}^{2}\left(A_{0}+r A_{1}\right)\right]=0 \\
3 a^{2} k r A_{1}+v \rho c A_{1}+\beta A_{1}^{2}+3 \alpha A_{1}^{2}\left(A_{0}+r A_{1}\right)=0, \\
p\left[a^{2} k A_{1}\left(r^{2}+2 s p\right)+\gamma A_{1}-v c r A_{1} p+2 \beta A_{1}\left(A_{0}+r A_{1}\right)+3 A_{1} \alpha\left(s p A_{1}^{2}+\left(A_{0}+r A_{1}\right)^{2}\right)\right]=0, \\
a^{2} k A_{1}\left(r^{2}+2 s p\right)+\gamma A_{1}+v c r A_{1} \rho+2 \beta A_{1}\left(A_{0}+r A_{1}\right)+3 A_{1} \alpha\left(s p A_{1}^{2}+\left(A_{0}+r A_{1}\right)^{2}\right)=0, \\
\delta+\gamma\left(A_{0}+A_{1} r\right)+2 a^{2} k s r p A_{1}+\beta\left(2 p s A_{1}^{2}+\left(A_{0}+r A_{1}\right)^{2}\right)+\alpha\left(6 s A_{1}^{2} p\left(A_{0}+r A_{1}\right)+\left(A_{0}+r A_{1}\right)^{3}\right)=0 .
\end{array}\right. \\
\left\{\begin{array}{l}
v=p=0,6 a^{2} k s^{2} A_{2}+\beta=0, \\
7 A_{2} s^{2} a^{2} k\left[\left(A_{1}+2 r A_{2}\right)^{2}+2\left(A_{2}+6 A_{2}^{2}\left(A_{1}+2 r A_{2}\right)\right)\left(A_{0}+r A_{1}+A_{2} r^{2}\right)\right] \\
+\gamma A_{2}+\beta\left[\left(A_{1}+2 r A_{2}\right)^{2}+2 A_{2}\left(A_{0}+r A_{1}+A_{2} r^{2}\right)\right]=0, \\
a^{2} k s\left(A_{1}+2 r A_{2}\right)\left[s\left(A_{1}+2 r A_{2}\right)^{2}+2 A_{2}(1+3 s)\left(A_{0}+r A_{1}+A_{2} r^{2}\right)\right]+\gamma\left(A_{1}+2 r A_{2}\right)+\beta\left[2\left(A_{1}+2 r A_{2}\right)\left(A_{0}+r A_{1}+A_{2} r^{2}\right)\right]=0 \\
\delta+s^{2} a^{2} k\left(A_{0}+r A_{1}+A_{2} r^{2}\right)\left[\left(A_{1}+2 r A_{2}\right)^{2}+2 A_{2}\left(A_{0}+r A_{1}+A_{2} r^{2}\right)\right]+\left(A_{0}+r A_{1}+A_{2} r^{2}\right)\left[\gamma+\beta\left(A_{0}+r A_{1}+A_{2} r^{2}\right)\right]=0 .
\end{array}\right.
\end{gathered}
$$


Here,

$$
\begin{aligned}
& \beta=-c_{b} \rho_{b} \beta_{0} ; \gamma=c_{b} \rho_{b}\left(\beta_{0} T_{a}-\alpha_{0}\right) ; \\
& \delta=Q_{m}+Q_{p}+\alpha_{0} T_{a} c_{b} \rho_{b} .
\end{aligned}
$$

Condition $v=0$ means the generalized Riccati equation mapping method in the case of linear blood perfusion can be applied just for the initial temperature distribution and the steady-state temperature distribution.

\subsection{Coupling Method for Thermal Traveling-Wave Distribution in Biological Tissues}

In this section, we split Equation (1.14) into two ordinary differential equations which lead to a Riccati equation that will help us to find analytical solutions.

It is obvious that $u(z)$ will satisfy Equation (1.14) as soon as it verifies the following two equations

$$
\begin{gathered}
\frac{\mathrm{d} u}{\mathrm{~d} z}=\frac{c_{b} \rho_{b}\left(\beta_{0}-\gamma_{0} T_{a}\right)}{v \rho c} u^{2}-\frac{Q_{m}+Q_{p}+\alpha_{0} T_{a} c_{b} \rho_{b}}{v \rho c} \\
\frac{\mathrm{d}^{2} u}{\mathrm{~d} z^{2}}=\frac{\gamma_{0} c_{b} \rho_{b}}{a^{2} k} u^{3}+\frac{c_{b} \rho_{b}\left(\alpha_{0}-\beta_{0} T_{a}\right)}{a^{2} k} u
\end{gathered}
$$

One easily verifies that any solution $u(z)$ of Equation (1.21a) will satisfy equation

$$
\begin{aligned}
\frac{\mathrm{d}^{2} u}{\mathrm{~d} z^{2}}= & \frac{2 c_{b}^{2} \rho_{b}^{2}\left(\beta_{0}-\gamma_{0} T_{a}\right)^{2}}{v^{2} \rho^{2} c^{2}} u^{3} \\
& -\frac{2 c_{b} \rho_{b}\left(\beta_{0}-\gamma_{0} T_{a}\right)\left(Q_{m}+Q_{p}+\alpha_{0} T_{a} c_{b} \rho_{b}\right)}{v^{2} \rho^{2} c^{2}} u .
\end{aligned}
$$

Therefore, $u(z)$ will satisfy Equation (1.21b) if and only, if Equation (1.21b) coincides with Equation (1.22), that is, if and only, if $\gamma_{0} \neq 0$ and

$$
\begin{aligned}
& v^{2}=\frac{2 a^{2} k c_{b} \rho_{b}\left(p_{2}-p_{3} T_{a}\right)^{2}}{10^{4} p_{3} \rho^{2} c^{2}} \\
& \text { and } p_{3}=\frac{c_{b} \rho_{b} p_{2}\left(p_{2} T_{a}-p_{1}\right)}{10^{4}\left(Q_{m}+Q_{p}\right)+p_{1} T_{a} c_{b} \rho_{b}+c_{b} \rho_{b}\left(p_{2} T_{a}-p_{1}\right) T_{a}}
\end{aligned}
$$

Thus, if blood perfusion parameters $p_{1}, p_{2}$, and $p_{3}$ and the wave velocity $v$ satisfy condition (1.23), then any solution of Equation (1.21a) will be solution of Equation (1.14). Henceforth, we will focus ourselves on Equation (1.12a). When $\beta_{0}-\gamma_{0} T_{a} \neq 0$, Equation (1.21a) coincides with Riccati Equation (1.5) with $r=0$,

$$
p=-\frac{Q_{m}+Q_{p}+\alpha_{0} T_{a} c_{b} \rho_{b}}{v \rho c}, \text { and } s=\frac{c_{b} \rho_{b}\left(\beta_{0}-\gamma_{0} T_{a}\right)}{v \rho c} \text {. }
$$

Under condition $\beta_{0}-\gamma_{0} T_{a} \neq 0$, we obtain from particular and the general solutions of Equation (1.21a) the following solutions of Equation (1.1) with perfusion Equation (1.2)

$$
=\left\{\begin{array}{l}
T(x, t) \\
\pm \sqrt{\tilde{\alpha}_{0}} \tanh \left[\mp \frac{c_{b} \rho_{b}\left(\beta_{0}-\gamma_{0} T_{a}\right)}{v \rho c} \sqrt{\tilde{\alpha}_{0}}(a x-v t)+C_{0}\right], \\
\text { if } \tilde{\alpha}_{0}>0, \\
\frac{v \rho c}{v \rho c C_{0}-c_{b} \rho_{b}\left(\beta_{0}-\gamma_{0} T_{a}\right)(a x-v t)}, \\
\text { if } \tilde{\alpha}_{0}=0, \\
\pm \sqrt{-\tilde{\alpha}_{0}} \tan \left[ \pm \frac{c_{b} \rho_{b}\left(\beta_{0}-\gamma_{0} T_{a}\right)}{v \rho c} \sqrt{-\tilde{\alpha}_{0}}(a x-v t)+C_{0}\right], \\
\text { if } \tilde{\alpha}_{0}<0,
\end{array}\right.
$$

and

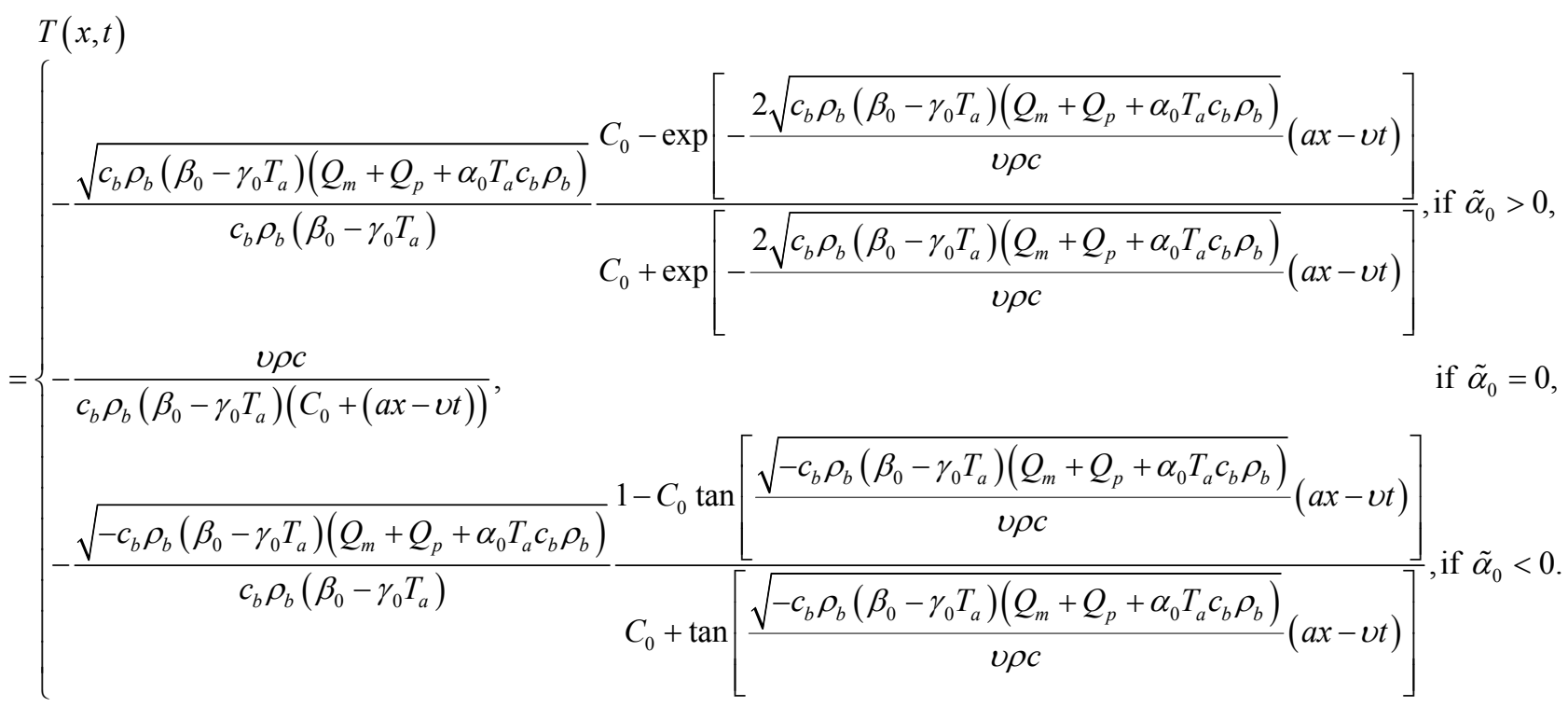


respectively. Here, $\tilde{\alpha}_{0}=\frac{Q_{m}+Q_{p}+\alpha_{0} T_{a} c_{b} \rho_{b}}{c_{b} \rho_{b}\left(\beta_{0}-\gamma_{0} T_{a}\right)}$ and $C_{0}$ is an arbitrary real constant.

\section{Results and Discussion}

In this section, we use the analytical solutions found in the previous section to discuss thermal traveling-wave distribution in biological tissues.

In the following calculations, the typical tissue properties are applied as given in [20]: $\rho=\rho_{b}=1000 \mathrm{~kg} / \mathrm{m}^{3}$, $c=c_{b}=4000 \mathrm{~J} / \mathrm{kg} \cdot{ }^{\circ} \mathrm{C}, \quad k=0.5 \mathrm{~W} / \mathrm{m} \cdot{ }^{\circ} \mathrm{C}, \quad T_{a}=37{ }^{\circ} \mathrm{C}$, $Q_{m}=420 \mathrm{~J} / \mathrm{m}^{3} \cdot \mathrm{s}$ for the healthy tissue and

$Q_{m}=4200 \mathrm{~J} / \mathrm{m}^{3} \cdot \mathrm{s}$ for the tumor. In our simulations, the skin surface and the body core are assumed to be defined at $x=0$ and $x=L$, respectively. As it is shown by several investigators $[44,45]$, the interior tissue temperature usually tends to a constant within a short distance such as $2-3 \mathrm{~cm}$. Therefore we will choose $L \in[2,3] \mathrm{cm}$ in our simulations. Following Partridge et al. [35], we will use for the temperature-dependent blood perfusion (1.2), the coefficients

$$
\alpha_{0}=p_{1} / 10000, \beta_{0}=p_{2} / 10000, \gamma_{0}=p_{3} / 10000,
$$

where for tumour $p_{1}$ takes values up to $7, p_{2}$ takes values up to 1.9 , and $p_{3}$ takes values up to 0.07 . In the case of linear blood perfusion, i.e., when $\gamma_{0}=p_{3}=0$, we will take $p_{1}$ and $p_{2}$ so that $\alpha_{0}$ and $\beta_{0}$ coincide with the coefficients of linear blood perfusion used in Ref. [36]. Because the variation of the constant spatial heating $Q_{r}$ can only affect the temperature magnitude at a given depth $x$, we will, without loss of generality, consider in all our simulations that $Q_{r}=0$ so that the initial temperature field for the basal state of biological bodies will coincide with the steady-state temperature fields.

We start our analysis with the case of linear temperature-dependent blood perfusion. Figure shows the initial/steady-state temperature distribution obtained in the case of linear temperature-dependent blood perfusion with the same baseline perfusion $\alpha_{0}=0.1$ and the linear perfusion coefficient $\beta_{0}=0.1,0.15,0.2$, respectively. The physical solutions of system (1.20) used for this figure are showed in Table 1. As one can see from Table 1, the physical solutions contain two parameters, $A_{2}$ and $r$. These two parameters can be defined in a concrete problem of heat transfer, for example, when the boundary conditions are given. For plots of Figure 1, we

Table 1. Solutions of system (1.20) obtained for the same baseline perfusion $\alpha_{0}$ and different linear coefficient $\beta_{0}$.

\begin{tabular}{|c|c|c|c|c|c|c|c|}
\hline$\alpha_{0}$ & $\beta_{0}$ & $\alpha$ & $A_{0}$ & $A_{1}$ & $A_{2}$ & $r$ & $\mathrm{~s}$ \\
\hline 0.1 & 0.01 & 514.4773608967789 & 52.822850449709875 & -0.8169156477513188 & $A_{2}$ & $r$ & 0.7097462384615433 \\
\hline 0.1 & 0.015 & 562.9344710809888 & 54.23750187274088 & -0.8526762513773855 & $A_{2}$ & $r$ & 0.7944327776573799 \\
\hline 0.1 & 0.02 & 613.8512954681446 & 55.007269523102615 & -0.869352875667146 & $A_{2}$ & $r$ & 0.8412424691561482 \\
\hline
\end{tabular}
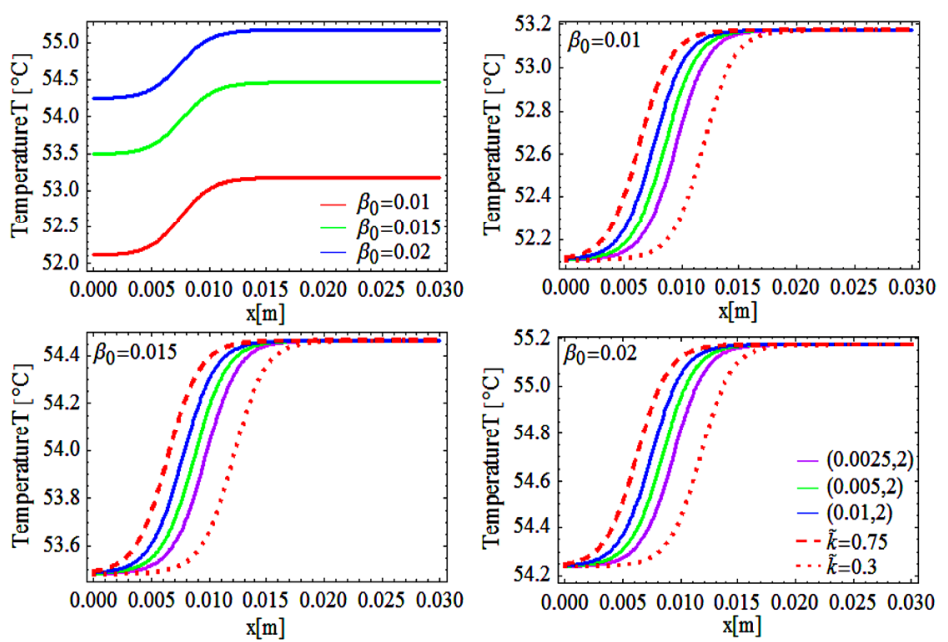

Figure 1. (Color online) Initial/steady-state temperature-dependent perfusion distributions for different linear coefficient of temperature dependence in the case of linear temperature-dependent blood perfusion. The perfusion level was dependent on local temperature with the same baseline perfusion $\alpha_{0}=0.1$ and a linear coefficient of temperature dependence shown in inset. Top left: Effect of linear coefficient of temperature dependence on temperature distribution; here, we have used $\left(C_{1}, C_{2}\right)=$ $(0.01,2)$ (see Equations (1.4)). Top and bottom right, bottom left: Spatial temperature distribution for different $\tilde{k}$ and different $\left(C_{1}, C_{2}\right)$ appearing in Equations (1.4) and (1.10), respectively; the same values of $\left(C_{1}, C_{2}\right)$ and $\tilde{k}$, shown in the bottom right plots have been used. The dashed and dotted curves show the temperature distribution obtained with the help of the nonlinear superposition (1.4). 
have used $A_{2}=0.1$ and $r=1$. The $3 \mathrm{~cm}$ long tissue has being used. The top left plot shows the spatial distribution of temperature for different values of perfusion $\beta_{0}$. As expected, decreased perfusion causes a decline in local temperature. Each of other three figures shows for the same $\beta_{0}$ temperature distribution obtained for different parameters $C_{1}$ and $C_{2}$ appearing in Equation (1.10) and different parameter $\stackrel{k}{k}$ of nonlinear superposition (1.4). The dashed and dotted curves show the temperature distribution obtained with the help of the nonlinear superposition (1.4); here, we have used three different $\left(C_{1}, C_{2}\right)$ to form the solutions for the superposition. As one can easily see from these three plots, different parameters $\left(C_{1}, C_{2}\right)$ give the same temperature at surface of the skin and at the body core. The top right and bottom left and right plots show that one may use the superposition parameter $\tilde{k}$ to manage (increase or de- crease) temperature of the biological tissues.

Let us discuss the case of a quadratic temperaturedependent blood perfusion. We will focus our attention on the case $v \neq 0$ and $p=0$, that is, the case of thermal traveling-wave distribution. We will investigate the effect of linear and quadratic coefficients of temperature dependence on temperature distribution, and then, show how one may used the parameter of the nonlinear superposition formula of Riccati equation to control the temperature in time and space.

Figure 2 shows the effect of the quadratic coefficient of temperature dependence on temperature distribution. For this figure, we have used the perfusion coefficients (1.26) with $\left(p_{1}, p_{2}, p_{3}\right)=(3,0.5,0.01),(3,0.5,0.02)$, $(3,0.5,0.03)$. The $3 \mathrm{~cm}$ long tissue has being used. For these values of $p_{1}, p_{2}$, and $p_{3}$, physical solutions of system (1.17) are given in Table 2. For these physical
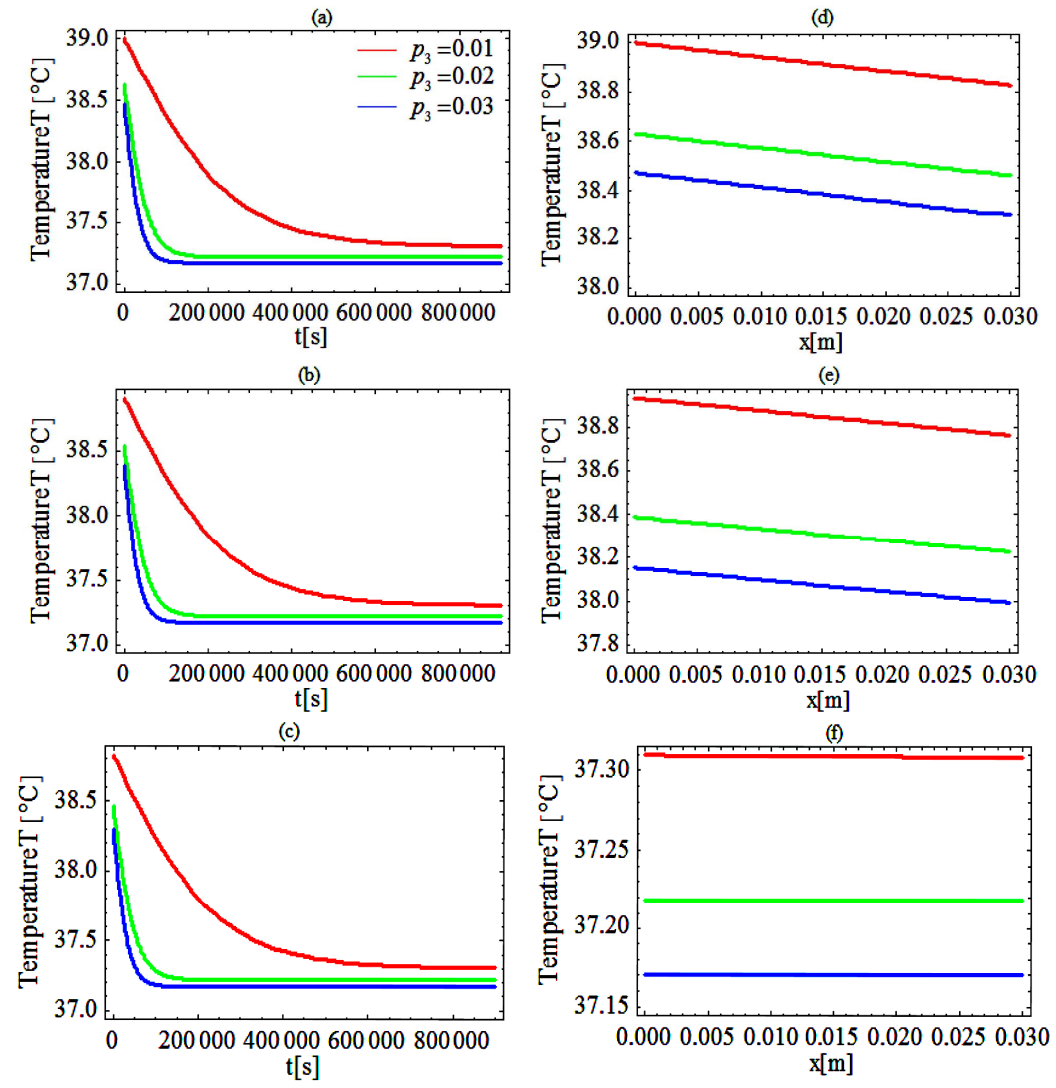

Figure 2. (Color online) Temporal and spatial temperature-dependent perfusion distributions: Effect of the quadratic coefficient of temperature dependence on temperature distribution. The left and right plots respectively show the temporal and spatial temperature distribution at different distance $x$ from skin surface and at different time $t$. Plots (a), (b), and (c) show the temperature distribution at skin surface, at distance $x=0.015 \mathrm{~m}$, and at the body core, respectively. Plots (d), (e), and (f) show the initial temperature distribution, the temperature distribution at time $t=10000 \mathrm{~s}$, and the steady-state temperature distribution, respectively. For all these plots, we have used solution (1.4)) with $\left(C_{1}, C_{2}\right)=(1,1)$. Here, we have used $p_{1}=3, p_{2}=$ 0.5, and different $p_{3}$ shown in plot (a) (see Equation (1.26)). We have used $a=0.20000000000000004, v=$ $-\mathbf{2 . 2 7 0 4 7 6 6 0 4 2 8 0 4 4 3 6} \times 10^{-7}, A_{0}=-47.48257513976442$, and $r=847.8483254334188$ for $p_{3}=0.01 ; a=0.28284271247461906, v$ $=-1.2284805279005027 \times 10^{-6}, A_{0}=-33.31293218373712$, and $r=705.3092927447257$ for $p_{3}=0.02 ;$ and $a=$ $0.3464101615137755, v=-1.9254281349374016 \times 10^{-6}, A_{0}=-27.89371450273014$, and $r=650.6425537226606$ for $p_{3}=0.03$. Other parameters used for the realization of these plots are given in the text. 
Table 2. Solutions of system (1.17) obtained for the same parameters $p_{1}$ and $p_{2}$ and different parameter $p_{3}:(a)\left(p_{1}, p_{2}, p_{3}\right)=(3$, $0.5,0.01) ;(\mathrm{b})\left(p_{1}, p_{2}, p_{3}\right)=(3,0.5,0.02) ;(c)\left(p_{1}, p_{2}, p_{3}\right)=(3,0.5,0.01)$.

(a)

\begin{tabular}{|c|c|c|c|c|c|}
\hline$\alpha$ & $v$ & $A_{0}$ & $A_{1}$ & $r$ & $s$ \\
\hline 0.20000000000000004 & 0.000025208402102574534 & 122.08708994691935 & $\mathrm{~A}_{1}$ & -847.8483254334188 & $s$ \\
\hline 0.20000000000000004 & $-2.737888943585129 \times 10^{-7}$ & 32.15753184033524 & $\mathrm{~A}_{1}$ & -748.0698964306056 & $s$ \\
\hline 0.20000000000000004 & -0.00002271588578727669 & -117.45644744578587 & $\mathrm{~A}_{1}$ & 748.0698964306056 & $S$ \\
\hline 0.20000000000000004 & $-2.2704766042804436 \times 10^{-7}$ & -47.48257513976442 & $\mathrm{~A}_{1}$ & 847.8483254334188 & $S$ \\
\hline
\end{tabular}

(b)

\begin{tabular}{|c|c|c|c|c|c|}
\hline$\alpha$ & $v$ & $A_{0}$ & $A_{1}$ & $r$ & $s$ \\
\hline 0.28284271247461906 & 0.00004109007703678306 & 107.748926365208 & $\mathrm{~A}_{1}$ & -705.3092927447257 & $s$ \\
\hline 0.28284271247461906 & $-1.2284805279005027 \times 10^{-6}$ & -33.31293218373712 & $\mathrm{~A}_{1}$ & 705.3092927447257 & $s$ \\
\hline
\end{tabular}

(c)

\begin{tabular}{cccccc}
\hline$\alpha$ & $v$ & $A_{0}$ & & $A_{1}$ & $r$ \\
\hline 0.3464101615137755 & 0.00005663240170010208 & 102.23479624180199 & $\mathrm{~A}_{1}$ & -650.6425537226606 & $s$ \\
0.3464101615137755 & $-1.9254281349374016 \times 10^{-6}$ & -27.89371450273014 & $\mathrm{~A}_{1}$ & 650.6425537226606 & $s$ \\
\hline
\end{tabular}

solutions, $A_{1}$ and $s$ appear as free parameters and can be particularized with the help of additional condition of the bio-heat transfer problem such as boundary conditions. For all plots of Figure 2, we have used $A_{1}=0.1$ and $s=-25$.

The left and right plots of Figure 2 respectively show the temporal and the spatial distribution of temperature at different distance $x$ from the skin surface and at different time $t$ for different values of $p_{3}$ (i.e., for different values of the quadratic coefficient of temperature dependence of blood perfusion). As expected, increased perfusion causes a decline in local temperature. As it is seen from the left plots, the temperature of the tissue at each distance $x$ from the skin surface decreases and approaches the steady-state temperature. It is also seen that with smallest $p_{3}$, the tissue temperature will quickly reach a steady state. As seen in right plots, tissue temperature decreases as one goes from the skin surface to body core.

To investigate the effect of linear coefficient of temperature dependence of the blood perfusion on temperature distribution, we fix $p_{1}$ and $p_{3}$, and vary $p_{2}$ as follows: $\left(p_{1}, p_{2}, p_{3}\right)=(7,1,0.07),(7,1.2,0.07)$,

$(7,1.4,0.07)$. For these values of $p_{1}$ and $p_{3}$, and $p_{2}$, physical solutions of system (1.17) are shown if Table 3. This table shows two free parameters, $A_{1}$ and $s$ which can be determined by using additional condition imposed on Equation (1.1) with potential (1.2).

Figure 3 shows the temporal (left plots) and the spatial (right plots) temperature distribution for a $3 \mathrm{~cm}$ long tissue. The plots of this figure have been generated with the help of Riccati solution (1.10). As solutions of system (1.17), we have used data from the first row of (a), (b), and (c) in Table 3, with $A_{1}=0.1$ and $s=-6$. As constants of solution (1.10), we have used $C_{1}=-17$ and $C_{2}=0.2$. As seen in plots (a) obtained with $x=0$, increased perfusion causes a decline in the temperature of skin surface. Plots (b) and (c) show that inside the tissue (when $x>0$ ), for a short period of heating, decreased perfusion causes a decline in local temperature. As one tends to the steady-state, higher perfusion gives lower local temperature. From plots (d) and (f) which respectively show the initial temperature distribution and the steady-state temperature fields, it is seen that increased perfusion causes an increase in the initial and steady-state local temperature of tissue.

Dashed and dotted curves of Figure 4 show the temperature distribution obtained with the help of nonlinear superposition formula (1.4) of Riccati equation. Other three curves of this figure show the temperature distribution obtained with the use of solution (1.10) after particularizing parameters $C_{1}$ and $C_{2}$; we have used the same parameter $C_{2}=0.2$ and three different $C_{1}=17$, 20,23 . To generate the solutions of the superposition, we have used $p_{1}=7, p_{2}=1$, and $p_{3}=0.07$ and the following solution of system (1.17): $\alpha=$

$0.5291502622129182, v=0.00012855305358758568, A_{0}$ $=100.49771736484482, r=-634.2109797960809, A_{1}=$ $0.1, r=-634.2109797960809, \quad p=0$, and $s=4$. Plots (a) and (b) show respectively the temperature of skin surface and body core as function of time $t$. Plots (c) and (d) depict the temperature curves as function of skin depth $x$ at time $t=0 \mathrm{~s}$ and time $t=90 \mathrm{~s}$. The four plots of Figure 4 show that one can easily readjust (increase or decrease) the temperature of the biological tissues, just by varying parameter $\tilde{k}$ of the nonlinear superposition formula (1.4) of Riccati equation.

Let us now validate solutions (1.24) and (1.25). This solution presents some restriction on blood perfusion (1.2). Indeed, $p_{1}, p_{2}$, and $p_{3}$ appearing in Equation (1.26) must satisfy the second equality in Equation (1.23). With this restriction, it is not possible to separately 
Table 3. Solutions of system (1.17) obtained for the same parameters $p_{1}$ and $p_{3}$ and different linear parameter $p_{2}$ : (a) $\left(p_{1}, p_{2}, p_{3}\right)$ $=(7,1,0.07) ;(\mathrm{b})\left(p_{1}, p_{2}, p_{3}\right)=(7,1.2,0.07) ;(\mathrm{c})\left(p_{1}, p_{2}, p_{3}\right)=(7,1.4,0.07)$.

(a)

\begin{tabular}{|c|c|c|c|c|c|}
\hline$\alpha$ & $v$ & $A_{0}$ & $A_{1}$ & $r$ & $s$ \\
\hline 0.5291502622129182 & 0.00012855305358758568 & 100.49771736484482 & $A_{1}$ & -634.2109797960809 & $s$ \\
\hline 0.5291502622129182 & $-4.631252169591368 \times 10^{-6}$ & -26.34447859437136 & $A_{1}$ & 634.2109797960809 & $s$ \\
\hline
\end{tabular}

(b)

\begin{tabular}{|c|c|c|c|c|c|}
\hline$\alpha$ & $v$ & $A_{0}$ & $A_{1}$ & $r$ & $s$ \\
\hline 0.5291502622129182 & 0.00013226855259313876 & 102.1353600675383 & $A_{1}$ & -650.6257480827784 & $s$ \\
\hline 0.5291502622129182 & $-4.36285450424477 \times 10^{-6}$ & -27.989789549017377 & $A_{1}$ & 650.6257480827784 & $s$ \\
\hline
\end{tabular}

(c)

\begin{tabular}{|c|c|c|c|c|c|}
\hline$\alpha$ & $v$ & $A_{0}$ & $A_{1}$ & $r$ & $s$ \\
\hline 0.5291502622129182 & 0.00013594278617606382 & 103.73333731695307 & $A_{1}$ & -666.6402113289303 & $s$ \\
\hline 0.5291502622129182 & $-4.05165820301162 \times 10^{-6}$ & -29.594704948832998 & $A_{1}$ & 666.6402113289303 & $s$ \\
\hline
\end{tabular}
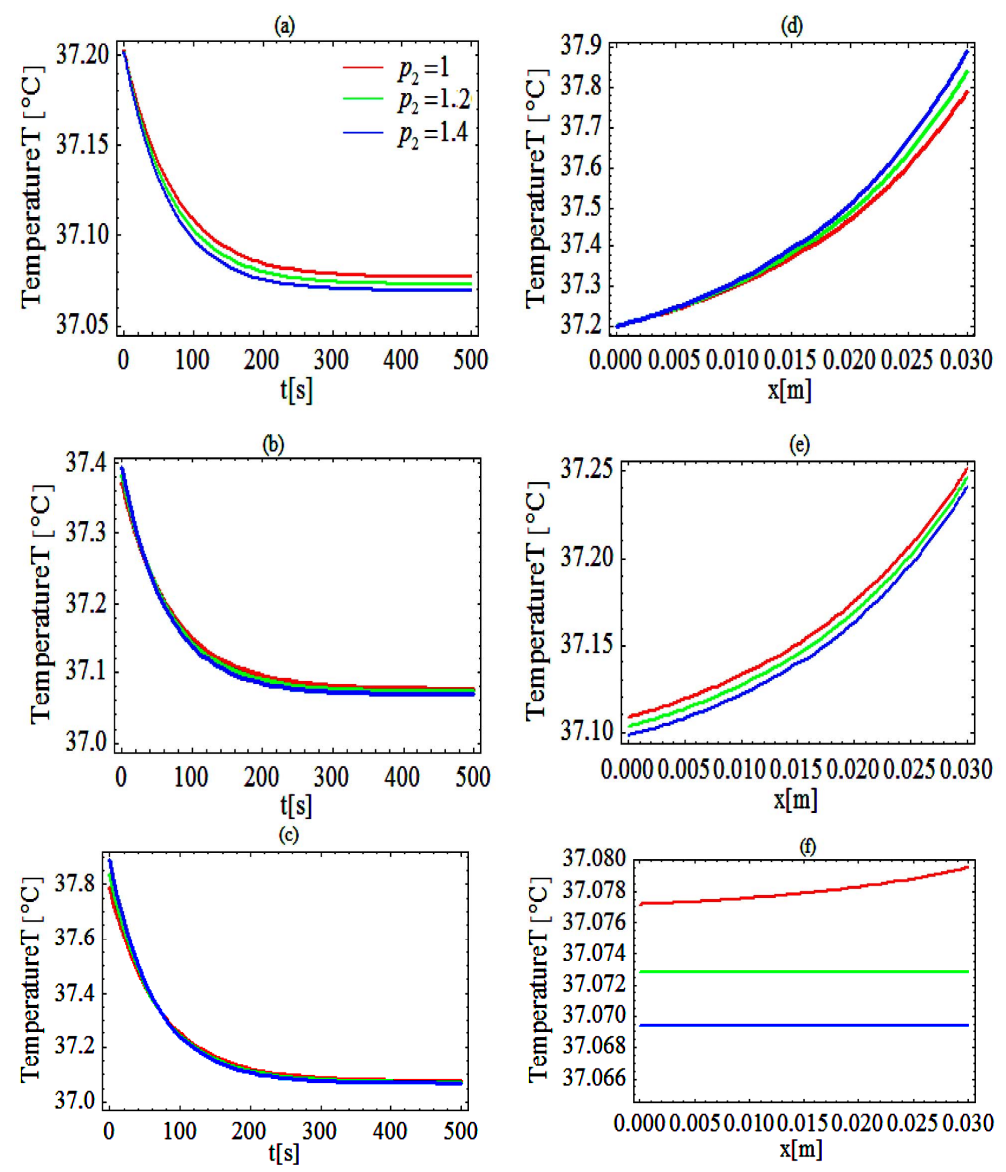

Figure 3. (Color online) Temporal and spatial temperature-dependent perfusion distributions: Effect of the linear coefficient of temperature dependence on temperature distribution. The left and right plots respectively show the temporal and spatial temperature distribution at different distance $x$ from skin surface and at different time $t$. Plots (a), (b), and (c) show the temperature distribution at skin surface, at distance $x=0.015 \mathrm{~m}$, and at the body core, respectively. Plots (d), (e), and (f) show the initial temperature distribution, the temperature distribution at time $t=100 \mathrm{~s}$, and the steady-state temperature distribution, respectively. For all these plots, we have used solution (1.4)) with $\left(C_{1}, C_{2}\right)=(-17,0.2)$. Here, we have used $p_{1}=7, p_{3}=$ 0.07, and different $p_{2}$ shown in plot (a) (see Equation (1.26)). We have used $a=0.5291502622129182, v=$ $0.00012855305358758568, A_{0}=100.49771736484482$, and $r=-634.2109797960809$ for $p_{2}=1 ; a=0.5291502622129182, v=$ $0.00013226855259313876, A_{0}=102.1353600675383$, and $r=-650.6257480827784$ for $p_{2}=1.2 ;$ and $a=0.5291502622129182, v$ $=0.00013594278617606382, A_{0}=103.73333731695307$, and $r=-666.6402113289303$ for $p_{2}=1.4$. Other parameters used for the realization of these plots are given in the text. 

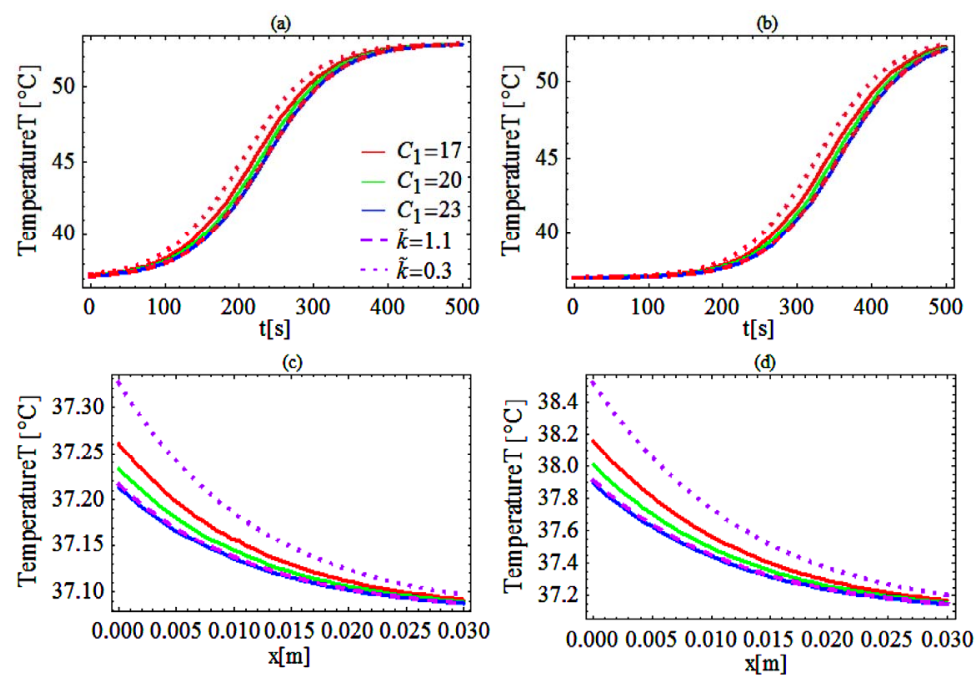

Figure 4. (Color online) Temperature management via the parameter $\tilde{\boldsymbol{k}}$ of nonlinear superposition formula of Riccati equation. (a), (b): Temperature of skin surface and temperature of the body core as a function of time. (c), (d): Temperature distribution as a function of skin depth $x$ for two different time points $t=0 \mathrm{~s}$ and $t=90 \mathrm{~s}$. Dashed and dotted curves show the temperature distribution obtained with the help of the nonlinear superposition formula (1.4) with $\tilde{k}=1.1$ (dashed curve) and $\tilde{k}=\mathbf{0 . 3}$ (dotted curve). Solution (1.10) of Riccati equation has been used with to generate the three solutions of the superposition; for these three solutions, we used in Equation (1.10) $C_{2}=0.2$ and $C_{1}$ shown in plot (a). The values of other parameters and constants are given in the text.
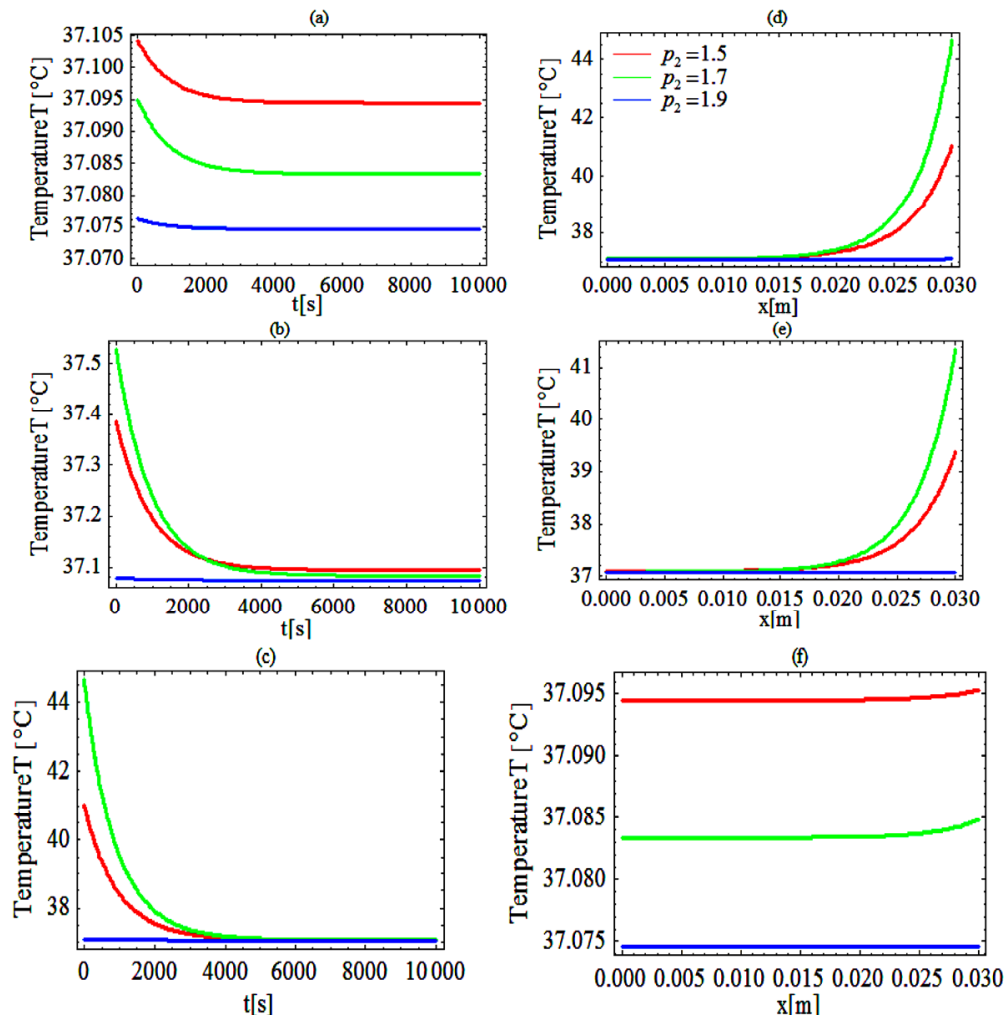

Figure 5. (Color online) Thermal traveling-wave distribution via either analytical solution (1.24) or (1.25).(a), (b), (c): Temporal temperature distribution at different skin depths $x=0.009 \mathrm{~m}, 0.021 \mathrm{~m}, 0.03 \mathrm{~m}$, respectively. (d), (e), (f): Temperature distribution as a function of skin depth at different times $t=0 \mathrm{~s}, 500 \mathrm{~s}, 8000 \mathrm{~s}$, respectively. The perfusion level was dependent on local temperature with the same baseline perfusion $\alpha_{0}=5 \times 10^{-4}$ and different linear coefficient of temperature dependence $\beta_{0}=10^{-4} p_{2}$, where parameter $p_{2}$ is in inset. The quadratic coefficient of temperature dependence used in this figure is $\gamma_{0}$ $=10^{-4} p_{3}$, where $p_{3}$ is defined by Equation (1.23). The values of other parameters and constants are given in the text. 
investigate the effect of the linear and quadratic coefficients of temperature dependence on temperature distribution. In fact, $p_{3}$ appears in Equation (1.23) as a function of $p_{2}$. We will work with $p_{1}=5$ and different values of $p_{2}$ and, consequently, different value of $p_{3}$. Figure 5 shows the thermal traveling-wave distribution obtained with the use of solution (1.24) with different $p_{2}: p_{2}=1.5,1.7,1.9$. Inserting these values of $p_{2}$ and $p_{1}=5$ in Equation (1.23), we obtain $p_{3}=0.0367006$, $0.0421037,0.0475073$, respectively. Thus, $p_{3}$ increases as $p_{2}$ increases. For all the plots of this figure, we have used $a=0.1$ and $C_{0}=-10^{-5}$. The left plots show the temperature distribution as a function of time $t$ at different depths $x=0.009 \mathrm{~m} \mathrm{(a),} x=0.021 \mathrm{~m} \mathrm{(b),} \mathrm{and}$ $x=L_{0}=0.03 \mathrm{~m}$. The right plots show the spatial temperature distribution for different time $t=0 \mathrm{~s} \mathrm{(d),} t=500$ $\mathrm{s}$ (e), and $t=8000 \mathrm{~s}$ (f). From plot (a) which shows the temporal distribution of temperature close to skin surface, it is seen that near the skin surface, increased perfusion causes a decline in local temperature. Away from the skin surface, the temperature curves intersect before the time of the steady-state temperature (see plots (b) and (c)). This means that away from the skin surface and before the steady-state time, increased perfusion does not necessary cause a decline in local temperature. This last fact can also be observed in plots (d) and (e). As it is seen from left plots, the higher steady-state temperature is reached at the body core. Plot (f) shows that higher perfusion gives lower steady-state temperature at any depth $x$ of the tissue.

\section{Discussion and Conclusion}

Analytical traveling-wave solutions of the $(1+1)$ generalized Pennes Equation obtained using the extended generalized Riccati equation mapping method for the hyperbolic (rational in exponential functions), trigonometric and rational function types are presented in this paper. This method is characterized by the fact that the construction of three particular solutions of the considered equation leads to an infinity of solutions via the nonlinear superposition formula of Riccati equation. With the use of the obtained solutions, we have analytically investigated the thermal traveling-wave distribution in biological tissue through a bio-heat transfer model with linear and quadratic temperature-dependent blood perfusion. For linear temperature-dependent blood perfusion, the method can give only initial temperature distribution which is useful for numerical simulations, and the steady-state temperature distribution which is useful to set the initial and boundary conditions of bio-heat transfer problem $[46,47]$. In the case of quadratic temperature-dependent blood perfusion, the initial condition may be obtained by setting the propagating velocity $v$ to zero, while the steady-state temperature of the tissue can be obtained by tending the time $t$ to plus infinity. After the initial time $t=0$ and before the steady-state time $T_{S t}$ (time after which the temperature at depth $x$ is constant with respect to time $t$ ), the obtained analytical solutions are used for temperature prediction at any depth $x$ at any time $t$. Our analytical solutions allowed us to investigate the effect of both linear and quadratic coefficients of temperature dependence on temperature distribution. We mainly showed that the increase in perfusion via the increased in the linear coefficient causes a decline in local temperature. On the other hand, it has been found that decreased perfusion via the quadratic coefficient of temperature-dependence causes decline in interior local temperature (that is, away from the skin surface). The application of the nonlinear superposition formula of the Riccati equation is useful for temperature control in the tissue. The analytical solutions found in this paper can be used to predicate the evolution of the detailed temperature within the tissues during thermal therapy.

\section{REFERENCES}

[1] J. Liu, L. Zhu and X. L. Xu, "Studies on the Three-Dimensional Temperature Transients in the Canine Prostate during Transurethal Micorwave Thermal Therapy," Journal of Biomechanical Engineering, Vol. 122, No. 4, 2000, pp. 372-379. http://dx.doi.org/10.1115/1.1288208

[2] G. T. Martin, H. F. Bowman, W. H. Newman and E. G. Cravalho, "Thermal Model with Temperature Dependent Perfusion for the Hyperthermia Treatment of Benign Prostatic Hyperplasia," Advanced Heat and Mass Transfer, Vol. 18, 1991, pp. 33-37.

[3] R. Seip and E. S. Ebbini, "Studies on the Three-Dimensional Temperature Response to Heating Fields Using Diagnostic Ultrasound," IEEE Transactions on Biomedical Engineering, Vol. 42, No. 8, 1995, pp. 828-839. http://dx.doi.org/10.1109/10.398644

[4] S. Puccini, N. K. Bär, M. Bublat, T. Kahn and H. Busse, "Simulations of Thermal Tissue Coagulation and Their Value for the Planning and Monitoring of Laser-Induced Interstitial Thermotherapy (LITT)," Magnetic Resonance in Medicine, Vol. 49, No. 2, 2003, pp. 351-362. http://dx.doi.org/10.1002/mrm.10357

[5] A. M. Stoll, "Thermal-Properties of Human-Skin Related to Nondestructive Measurement of Epidermal Thickness," Journal of Investigative Dermatology, Vol. 69, 1977, pp. 328-332. http://dx.doi.org/10.1111/1523-1747.ep12507865

[6] A. M. Stoll, M. A. Chianta and J. R. Piergallini, "Thermal Conduction Effects in Human-Skin," Aviation, Space, and Environmental Medicine, Vol. 50, No. 8, 1979, pp. 778-787.

[7] E. Kengne, A. Lakhssassi, R. Vaillancourt and W. M. Liu, "Monitoring of Temperature Distribution in Living Biological Tissues via Blood Perfusion," The European Physical Journal Plus, Vol. 127, No. 89, 2012, 15p.

[8] A. Lakhssassi, E. Kengne and H. Semmaoui, "Investiga- 
tion of Nonlinear Temperature Distribution in Biological Tissues by Using Bioheat Transfer Equation of Pennes' Type," Natural Science, Vol. 2, No. 3, 2010, pp. 131-138. http://dx.doi.org/10.4236/ns.2010.23022

[9] E. Kengne, A. Lakhssassi and R. Vaillancourt, "Temperature Distributions for Regional Hypothermia Based on Nonlinear Bioheat Equation of Pennes Type: Dermis and Subcutaneous Tissues," Applied Mathematics, Vol. 3, No. 3, 2012, pp. 217-224.

http://dx.doi.org/10.4236/am.2012.33035

[10] F. C. Henriques and A. R. Moritz, "Studies of Thermal Injury, 1. The Conduction of Heat to and through Skin and the Temperatures Attained Therein. A Theoretical and Experimental Investigation," American Journal of Pathology, Vol. 23, No. 4, 1947, pp. 431-549.

[11] H. H. Pennes, "Analysis of Tissue and Arterial Blood Temperature in the Resting Human Forearm," Journal of Applied Physiology, Vol. 1, No. 2, 1948, pp. 93-122.

[12] J. A. J. Stolwijk and J. D. Hardy, "Temperature Regulation in Man-A Theoretical Study," Pflugers Archives, Vol. 291, No. 2, 1966, pp. 129-162. http://dx.doi.org/10.1007/BF00412787

[13] E. H. Wissler, "Comparison of Results Obtained from Two Mathematical Models-A Simple 14-Node Model and a Complex 250-Node Model," Journal of Physiology (Paris), Vol. 63, No. 3, 1970, pp. 455-458.

[14] A. Shitzer and R. C. Eberhart, "Heat Transfer in Medicine and Biology," Plenum Press, New York, 1985. http://dx.doi.org/10.1007/978-1-4684-8285-0

[15] M. J. Mantyla, J. Kuikka and A. Rekonnen, "A Regional Blood Flow in Human Tumors with Special Reference to the Effect of Radiotherapy," British Journal of Radiology, Vol. 49, 1976, pp. 335-338. http://dx.doi.org/10.1259/0007-1285-49-580-335

[16] J. A. Surrell, R. C. Alexander, S. D. Cohle Jr., F. R. Lovell and R. A. Wehrenberg, "Effects of Microwave Radiation on Living Tissues," Journal of Trauma-Injury Infection \& Critical Care, Vol. 27, No. 8, 1987, pp. 935-939. http://dx.doi.org/10.1097/00005373-198708000-00014

[17] Z.-S. Deng and J. Liu, "Parametric Studies on the Phase Shift Method to Measure the Blood Perfusion of Biological Bodies," Medical Engineering \& Physics, Vol. 22, No. 10, 2000, pp. 693-702.

http://dx.doi.org/10.1016/S1350-4533(01)00015-7

[18] Z.-S. Deng and J. Liu, "Mathematical Modelling of Temperature Mapping over Skin Surface and Its Implementation in Thermal Disease Diagnostics," Computers in Biology and Medicine, Vol. 34, No. 6, 2004, pp. 495-521. http://dx.doi.org/10.1016/S0010-4825(03)00086-6

[19] Z.-S. Deng and J. Liu, "Modelling of Multidimensional Freezing Problem during Cryosurgery by the Dual Reciprocity Boundary Element Method," Engineering Analysis with Boundary Elements, Vol. 28, No. 2, 2004, pp. 97-108. http://dx.doi.org/10.1016/S0955-7997(03)00128-0

[20] J. Liu and L. S. Xu, "Boundary Information Based Diagnostics on the Thermal States of Biological Bodies," International Journal of Heat and Mass Transfer, Vol. 43,
No. 16,2000 , pp. $2827-2839$. http://dx.doi.org/10.1016/S0017-9310(99)00367-1

[21] E. H. Wissler, "Pennes' 1948 Paper Revisited," Journal of Applied Physiology, Vol. 85, No. 1, 1998, pp. 35-41.

[22] R. B. Roemer, B. R. Paliwal, F. W. Hetzel and M. W. Dewhirst, "Heat Transfer in Hyperthermia Treatments: Basic Principles and Applications," In: B. R. Paliwal, F. W. Hetzel and M. W. Dewhirst, Eds., Biological Physical and Clinical Aspects of Hyperthermia, American Institute in Physics, New York, 1988, pp. 210-242.

[23] S. Acharya, D. B. Gurung and V. P. Saxena, "Effect of Metabolic Reactions on Thermoregulation in Human Males and Females Body," Applied Mathematics, Vol. 4, No. 5A, 2013, pp. 39-48. http://dx.doi.org/10.4236/am.2013.45A005

[24] D. E. Haines and D. D. Watson, "Tissue Heating during Radiofrequency Catheter Ablation: A Thermodynamic Model and Observations in Isolated Perfused and Superfused Canine Right Ventricular Free Wall," Pacing and Clinical Electrophysiology, Vol. 12, No. 6, 1989, pp. 962976. http://dx.doi.org/10.1111/j.1540-8159.1989.tb05034.x

[25] K. R. Diller, "Modeling of Bioheat Transfer Processes at High and Low Temperatures," Advances in Heat Transfer, Vol. 22, 1992, pp. 157-167. http://dx.doi.org/10.1016/S0065-2717(08)70345-9

[26] R. W. Y. Habash, R. Bansal, D. Krewski and H. T. Alhafid, "Thermal Therapy, Part IV: Electromagnetic and Thermal Dosimetry," Critical Reviews in Biomedical Engineering, Vol. 35, No. 1-2, 2007, pp. 123-182. http://dx.doi.org/10.1615/CritRevBiomedEng.v35.i1-2.30

[27] E. Erdmann, J. Lang and M. Seebass, "Optimization of Temperature Distributions for Regional Hyperthermia Based on a Nonlinear Heat Equation," In: K. Diller, Ed., Biotransport: Heat and Mass Transfer in Living Systems, 1998, pp. 36-46.

[28] K. R. Foster, A. Lozano-Nieto, P. J. Riu and T. S. Ely, "Heating of Tissues by Microwaves: A Model Analysis," Bioelectromagnetics, Vol. 19, No. 7, 1998, pp. 420-428. http://dx.doi.org/10.1002/(SICI)1521-186X(1998)19:7<4 20::AID-BEM3 $>3.0 . \mathrm{CO} ; 2-3$

[29] P. J. Riu, K. R. Foster, D. W. Blick and E. R. Adair, “A Thermal Model for Human Thresholds of MicrowaveEvoked Warmth Sensations," Bioelectromagnetics, Vol. 18, No. 8, 1997, pp. 578-583. http://dx.doi.org/10.1002/(SICI)1521-186X(1997)18:8<5 78::AID-BEM6>3.0.CO;2-\#

[30] H. F. Bowman, E. G. Cravalho and M. Woods, "Theory, Measurement, and Application of Thermal Properties of Biomaterials," Annual Review of Biophysics and Bioengineering, Vol. 4, 1975, pp. 43-80. http://dx.doi.org/10.1146/annurev.bb.04.060175.000355

[31] G. T. Martin, H. F. Bowman and W. H. Newman, "Basic Element Method for Computing the Temperature Field during Hyperthermia Therapy Planning," Adv Bio Heat Mass Transf, Vol. 231, 1992, pp. 75-80.

[32] C. W. Song, A. Lokshina, J. G. Rhee, M. Patten and S. H. Levitt, "Implication of Blood Flow in Hyperthermic 
Treatment of Tumors," IEEE Transactions on Biomedical Engineering, Vol. 31, No. 1, 1984, pp. 9-16. http://dx.doi.org/10.1109/TBME.1984.325364

[33] J. Lang, B. Erdmann and M. Seebass, "Impact of Nonlinear Heat Transfer on Temperature Control in Regional Hyperthermia," IEEE Transactions on Biomedical Engineering, Vol. 46, No. 9, 1999, pp. 1129-1138. http://dx.doi.org/10.1109/10.784145

[34] C. R. Davies, G. M. Saidel and H. Harasaki, "Sensitivity Analysis of One-Dimensional Heat Transfer in Tissue with Temperature-Dependent Perfusion," Journal of Biomechanical Engineering, Vol. 119, No. 1, 1997, pp. 7780. http://dx.doi.org/10.1115/1.2796068

[35] W. P. Partridge and L. C. Wrobel, "A Coupled Dual Reciprocity BEM/Genetic Algorithm for Identification of Blood Perfusion Parameters," International Journal of Numerical Methods for Heat \& Fluid Flow, Vol. 19, No. 1, 2009, pp. 25-38. http://dx.doi.org/10.1108/09615530910922134

[36] T. R. Gowrishankar, D. A. Stewart, G. T. Martin and J. C. Weaver, "Transport Lattice Models of Heat Transport in Skin with Spatially Heterogeneous, Temperature-Dependent Perfusion," BioMedical Engineering on Line, Vol. 3, 2004, pp. 1-17.

[37] S. Lie and G. Scheffers, "Vorlesungenüber Continuierliche Gruppen mil Geometrischen und Anderen Anwend," Chelsea Publishing Company, New York, 1967.

[38] J. D. Cole, “On a Quasilinear Parabolic Equation Occurring in Aerodynamics," Quarterly of Applied Mathematics, Vol. 9, 1951, pp. 225-236.

[39] E. Hopf, "The Partial Differential Equation $u_{t}+u u_{x}=$ $u_{x x}$, "Communications on Pure and Applied Mathematics, Vol. 3, No. 3, 1950, pp. 201-230. http://dx.doi.org/10.1002/cpa.3160030302
[40] E. L. Ince, “Ordinary Differential Equations," Dover Publ., New York, 1956.

[41] Ph. Hartman, "Ordinary Differential Equations," John Wiley, New York, 1964.

[42] S. Zhu, "The Generalizing Riccati Equation Mapping Method In Non-Linear Evolution Equation: Application To (2+1)-Dimensional Boiti-Leon-Pempinelle Equation," Chaos, Solitons and Fractals, Vol. 37, No. 5, 2008, pp. 1335-1342. http://dx.doi.org/10.1016/j.chaos.2006.10.015

[43] H. S. Carslaw and J. C. Jaeger, "Conduction of Heat in Solids," Second Edition, Oxford University Press, Oxford, 1959.

[44] J. Liu and L. X. Xu, "Estimation of Blood Perfusion Using Phase Shift in Temperature Response to Sinusoidal Heating at the Skin Surface," IEEE Transactions on Biomedical Engineering, Vol. 46, No. 9, 1999, pp. 1037 1043. http://dx.doi.org/10.1109/10.784134

[45] S. Weinbaum, L. M. Jiji and D. E. Lemons, "Theory and Experiment for the Effect of Vascular Microstructure on Surface Tissue Heat Transfer-Part I: Anatomical Foundation and Model Conceptualization," Journal of Biomechanical Engineering, Vol. 106, No. 4, 1984, pp. 321-330. http://dx.doi.org/10.1115/1.3138501

[46] F. Rossi, R. Pini and L. Menabuoni, "3D Simulation and Experimental Comparison of Temperature Dynamics in Laser Welded Cornea," Proceedings of the COMSOL Users Conference, Milano, 2006.

[47] M. J. Rivera, J. A. Lopez Molina, M. Trujillo, V. Romero-Garcia and E. J. Berjano, "Analytical Validation of Comsol Multiphysics for Theoretical Models of Radiofrequency Ablation Including the Hyperbolic Bioheat Transfer Equation," Engineering in Medicine and Biology Society $(E M B C)$, Annual International Conference of the IEEE, 2010, pp. 3214-3217. 